\title{
Processing of probabilistic information in weight perception and motor prediction
}

\author{
Leif Trampenau $^{1}$ - Thilo van Eimeren ${ }^{2} \cdot$ Johann Kuhtz-Buschbeck $^{3}$
}

Published online: 29 December 2016

(C) The Psychonomic Society, Inc. 2016

\begin{abstract}
We studied the effects of probabilistic cues, i.e., of information of limited certainty, in the context of an action task (GL: grip-lift) and of a perceptual task (WP: weight perception). Normal subjects $(\mathrm{n}=22)$ saw four different probabilistic visual cues, each of which announced the likely weight of an object. In the GL task, the object was grasped and lifted with a pinch grip, and the peak force rates indicated that the grip and load forces were scaled predictively according to the probabilistic information. The WP task provided the expected heaviness related to each probabilistic cue; the participants gradually adjusted the object's weight until its heaviness matched the expected weight for a given cue. Subjects were randomly assigned to two groups: one started with the GL task and the other one with the WP task. The four different probabilistic cues influenced weight adjustments in the WP task and peak force rates in the GL task in a similar manner. The interpretation and utilization of the probabilistic information was critically influenced by the initial task. Participants who started with the WP task classified the four probabilistic cues into four distinct categories and applied these categories to the subsequent GL task. On the other side, participants who
\end{abstract}

Electronic supplementary material The online version of this article (doi:10.3758/s13414-016-1266-5) contains supplementary material, which is available to authorized users.

Leif Trampenau

1_trampenau@hotmail.com

1 Klinik für Neurologie, Universitatsklinikum Schleswig-Holstein Campus Kiel, Kiel, Germany

2 Department of Nuclear, Medicine, Universitätsklinikum Köln, Köln, Germany

3 Physiologisches Institut, Christian-Albrechts-Universität Kiel, Kiel, Germany started with the GL task applied three distinct categories to the four cues and retained this classification in the following WP task. The initial strategy, once established, determined the way how the probabilistic information was interpreted and implemented.

Keywords Probabilities - Anticipation · Motor system · Weight perception $\cdot$ Perception-action

\section{Introduction}

Dexterous manual performance is characterized by predictive scaling of the forces applied by the fingers according to pertinent task demands. When an object is grasped and lifted with a precision grip, the vertical load force (lift force) overcomes the force of gravity, while the grip force (normal to the grip surfaces) must be large enough to prevent slipping of the object. During skilled performance, both forces increase in concert and are scaled predictively according to relevant object properties such as shape, weight, and texture of the grip surfaces (Johansson \& Westling, 1984). Accurate anticipatory scaling of the forces is undemanding when well-known objects with unchanging properties are handled (Gordon, Westling, Cole, $\&$ Johansson, 1993). Such force control ensures an efficient and critically damped lift and avoids initial force undershoots or overshoots, which would require corrections (Johansson \& Flanagan, 2009; Nowak \& Hermsdörfer, 2005). The curves of the grip and load force rates are approximately bell-shaped and typically reach their maxima before lift-off (Johansson $\&$ Westling, 1988). Because exact sensory information about the object's weight is not available until lift-off, the peak values of the force rates are considered to indicate preprogramed forces (Chouinard, Leonard, \& Paus, 2005; 
Jenmalm, Schmitz, Forssberg, \& Ehrsson, 2006; Nowak, Glasauer, \& Hermsdörfer, 2013).

When novel objects are grasped and lifted, the peak force rates are scaled to the expected weight, based on visual cues to object size, material, and density (Gordon et al., 1993; Baugh, Kao, Johansson, \& Flanagan, 2012; Buckingham, Cant, \& Goodale, 2009). Normal volunteers also quickly learn to utilize arbitrary sensory cues (e.g., symbols presented on a monitor, sounds), which unmistakably predict object weight or texture of the gripped surfaces, for an adequate preprograming of their grip and load forces (Ameli, Dafotakis, Fink \& Nowak, 2008; Cole \& Rotella, 2002). Such associative learning conceivably involves a close cooperation between perception and action. However, sensory information about object properties can be equivocal. For instance, the normal mapping between material and weight is violated when surface material and core material of an object differ, e.g., when a small brassfilled cube is covered with wood veneer (Ellis \& Lederman, 1999). When people repeatedly lift such an unusual object and then predict the weight of a larger object of similar appearance, sensorimotor memory from lifts of the "outlier object" interferes with well-learned prior associations between material and density (Baugh et al., 2012). Hence, different internal models regarding the weight of an object can coexist, and predictive scaling of the force depends on the respective probability of each model.

Contemporary research considers movement planning from the viewpoint of decision-making under risk and applies the same mathematical framework that formalizes decisionmaking in economics and psychology (Nagengast, Braun, \& Wolpert, 2010; Wolpert \& Landy, 2012; Wu, Delgado, \& Maloney, 2009). So-called decisions under risk are made when participants have access to the probabilities associated with possible actions. In line with this approach, we recently examined the influence of explicit probabilistic advance information about object weight in a grip-lift (GL) task (Trampenau, Kuhtz-Buschbeck, \& van Eimeren, 2015). Three clearly discernible weights (medium, light, and heavy) were grasped and lifted. Before each lift, a visual cue provided probabilistic information about the forthcoming weight (e.g., $33.3 \%$ medium, $66.7 \%$ heavy) of the object, namely a moveable handle equipped with force transducers, whose weight was varied by a linear actuator.

The probabilistic cues systematically influenced peak grip and load force rates, as an index of predictive motor scaling. The same object of medium weight ( $800 \mathrm{~g}$ ) was grasped and lifted differently, depending on the expectation evoked by the probabilistic advance information. Cues that predicted a high likelihood of a weight differing from the medium value (800 g) had disproportionately stronger influence on predictive force scaling than cues that indicated a low likelihood of such a divergence, so that the anticipatory adaptations of the motor output seemed to overestimate high probabilities and to underestimate low probabilities. We interpreted this nonlinear effect as a distortion of probabilistic information on object weight during preparation of the grip-lift, as it has been demonstrated previously with other paradigms (Zhang \& Maloney, 2012).

To understand this pattern further, the present study investigated whether the perceptual system and the motor system utilize probabilistic information to an object's weight in a similar way. We devised a weight perception (WP) task, where participants adjusted the weight of the above-mentioned object (handle with force transducers) until the perceived heaviness was equal to what the participant believed would be the expected weight, based on the probabilistic cue. The WP task provided explicit information about the weight that is attributed to each probabilistic cue, which is not directly available from the force rate data of a grip-lift task (Trampenau et al., 2015). In a separate experiment, the same probabilistic cues were presented before the object was grasped and lifted (GL task). Following standardization of the results (z-transformation), we compared the effects of the probabilistic information on action initiation (GL task) and weight perception (WP task). Two groups of participants performed both tasks (WP and GL) in a different order to detect possible sequence effects.

The interpretation of probabilistic information can depend on the type of a task with its particular focus (Zhang \& Maloney, 2012). Similar effects of the probabilistic cues in both tasks (GL and WP) may indicate that perception and action rely on common abstract concepts in these experiments. By contrast, different effects of the probabilistic cues in both tasks may point to a dissociation of action and perception. Such dissociations have been shown previously, e.g., in the context of size-weight illusions for grip force scaling and weight perception (Flanagan \& Beltzner, 2000; Grandy \& Westwood, 2006; Chouinard, Large, Chang, \& Goodale, 2009). Comparing the use of probabilistic cues in the GL and WP tasks provides new insights into the way the perceptual and the motor systems process and implement probabilistic information about an object's weight.

\section{Methods}

Twenty-two right-handed healthy volunteers (10 men), age 25 \pm 5 years (mean, SD), were randomly assigned to two groups $A$ and $B(n=11$ participants per group of 5 men). This sample size was based on a power analysis using the maximum effect size $\left(\mathrm{d}_{\text {Cohen }}=1.781\right)$ of six effects from our previous grip-lift experiments (Trampenau et al., 2015), aiming for a power greater than 0.99167 with alpha $=0.00168$ (one-tailed). Group A started with the grip-lift (GL) task and afterwards performed the weight perception task (WP). Group B started with the latter task $\left(\mathrm{WP}_{1}\right)$, followed by the GL task, and finally 
performed the weight perception task once more $\left(\mathrm{WP}_{2}\right)$. All participants gave their informed consent to the experiments, which had been approved by the local ethics committee. They were naive to the purpose of the study.

\section{Grip-lift task}

The subjects sat on an adjustable chair at a table facing a monitor. The right forearm and wrist were oriented parallel to the sagittal plane and rested in a relaxed posture on a pad. The elbow joint angle was approximately $130^{\circ}$, and the hand was positioned in a semiprone posture with an ulnar adduction of the wrist joint of $\sim 10^{\circ}$ in the starting position. The grip object was essentially a moveable handle, consisting of a cuboid block attached to an adjustable lever (Fig. 1a). The length of the lever was set so that its rotational axis was aligned with the dorsopalmar axis of the radiocarpal joint. The distance between the axis of the lever and the center of the cuboid block varied between 12 and $15 \mathrm{~cm}$, depending on the size of the hand. The block had two flat vertical grip surfaces ( 4 x 4 $\mathrm{cm}$, spaced 3-cm apart) covered with sandpaper (grit size 600) and was equipped with custom-made nonmetallic force transducers (Dasch, Kiel, Germany). The angle between the block and lever was adjusted so that the grip surfaces were located close to the tips of the thumb and index finger in the starting position; for details see Supplementary Figure 1. Thus, the block could be grasped comfortably with a pinch grip between thumb and index finger, and it was lifted approximately $3-\mathrm{cm}$ high by a radial abduction of the wrist joint of $\sim 20^{\circ}$. The axis of the lever permitted a lifting movement in a vertical plane along a slightly curved movement path. The weight of the object was systematically changed from trial to trial during the experiment by a linear actuator (Servotube actuator

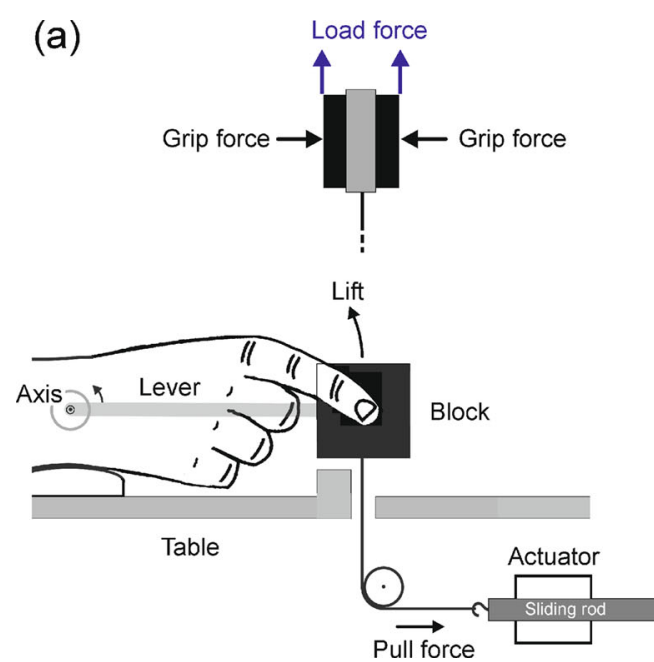

Fig. 1 Experimental set-up. (a) The object consisted of a block with force transducers attached to an adjustable lever, whose axis was aligned with the wrist joint. The block was grasped between thumb, index and middle finger and lifted along a slighty curved movement path. Object weight was set by a linear actuator, whose sliding rod
STA1116, Dunkermotoren, Essex, UK), which was hidden beneath the table. Its sliding rod was connected to the block via a nonexpandable string (Fig. 1a). Movements of the block were registered with an optical sensor connected to the rod at a sampling frequency of $50 \mathrm{~Hz}$. Grip and load force data were sampled with the same frequency. Original force curves are illustrated in supplementary Figure 2.

Three object weights were used throughout the GL task, namely $300 \mathrm{~g}$ (light), $800 \mathrm{~g}$ (medium), and $1400 \mathrm{~g}$ (heavy). Initially, the participants became familiar with these three typical weights, which were lifted ten times each in randomized order. Each weight was announced explicitly. Following the initial training, the four probabilistic visual cues were introduced and thoroughly explained. Each cue provided information about the likely object weight in the upcoming grip-lift trial (Fig. 1b). A blue rectangle could take four different positions in a black frame. The uppermost position (HM cue) indicated that the next object would be heavy in $66.7 \%$ and medium in $33.3 \%$ of the trials. The second position (MH cue) indicated that these percentages were reversed $(66.7 \%$ medium $/ 33.3 \%$ heavy). The third position (ML cue) indicated that object weight would be medium in $66.7 \%$ and light in $33.3 \%$ of the next trials, and the lowest position (LM cue) signified that these frequencies were reversed. Hence four probabilistic cues were used (LM, ML, MH, HM), each designating a fixed ratio of probabilities for two possible object weights.

All participants were encouraged to use these cues to prepare their grip-lift action. At the onset of each trial, a cue was shown on the monitor for one second (Fig. 2a). It then disappeared and emerged in smaller size beneath a target zone. This signaled that the object should, without long hesitation, be grasped and lifted by $\sim 3 \mathrm{~cm}$, thereby moving the cue into the target zone. A release-cue then requested to place the

(b)

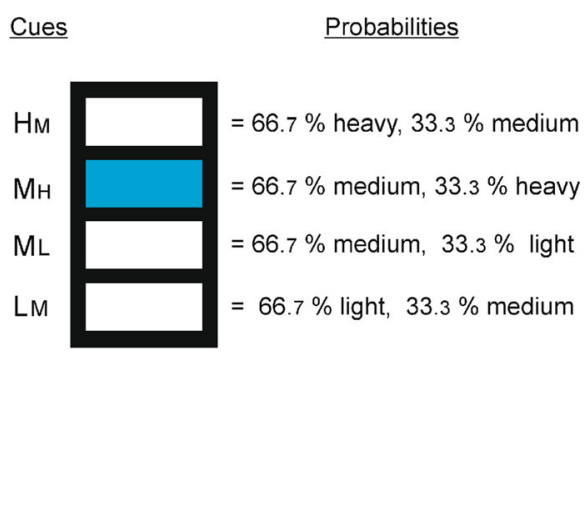

exerted programmable pull force via a non-elastic string that was attached to the lever. For details see supplementary figure I. (b) Probabilistic visual cues (here cue MH). Each position of the blue rectangle in the black frame signified the probabilities of two possible object weights. Four different cues were used (HM, MH, ML, LM) 
(a) Grip-lift task (GL)

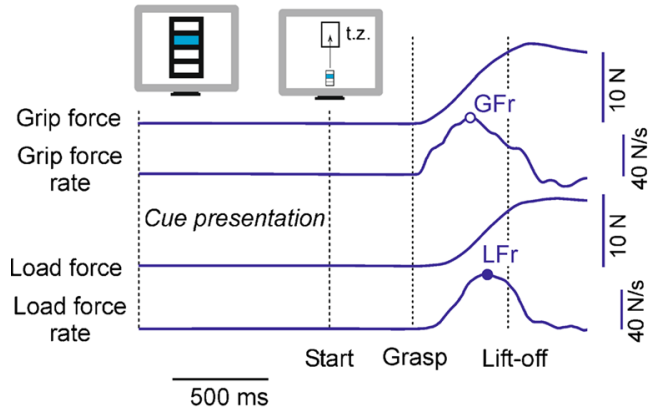

Fig. 2 Tasks. (a) In the grip-lift (GL), one probabilistic cue was shown for 1 second on a monitor, followed by a start cue with a target zone (t.z.). Then the object was grasped and lifted. Peak load force rate (LFr, filled blue circle) and peak grip force rate (GFr, open blue circle) are typically reached before lift-off; see also supplementary figure II for original data.

object back down and to release it. The next trial followed 3.5 seconds later. Each participant completed 150 consecutive grip-lift actions. Probabilistic visual cues announced the object weight in 120 trials. In another 30 trials, placed regularly throughout the experiment, object weight was announced unequivocally. All trials were performed in a pseudorandomized order that avoided possible systematic effects (Cole \& Rotella, 2002; Ameli et al., 2008; Nowak et al., 2013) of the preceding object weight. The light and the heavy object were lifted 40 times each, and the medium object was lifted 70 times. The grip-lift task lasted approximately $40 \mathrm{mi}-$ nutes (including instructions and initial training). Further methodical details have been published elsewhere (Trampenau et al., 2015).

\section{Weight perception task}

Participants of group B, who started with the WP task, were initially made acquainted with the three typical weights of the object (light: 300 g, medium: 800 g, heavy: 1,400 g), which were grasped and lifted ten times each in randomized order. Each weight was announced explicitly. Then the same four probabilistic cues as in the GL task were introduced and explained (see above), i.e., subjects were told that each cue signaled the probabilities $(66.7 \% / 33.3 \%)$ for two possible object weights. Participants of group A, who had already accomplished the GL task, skipped the initial familiarization phase and started directly with the WP task.

During each trial of this task one probabilistic cue was shown on the monitor (Fig. 2b). Participants were asked to associate the cue with a specific object weight, which matched the expected weight of the probabilistic prediction. For this, they lifted and held the object with a pinch grip (as in the GL task) of their right hand and then gradually changed its weight by pressing the arrow keys $(\downarrow \uparrow)$ of a computer keyboard with the left hand until the perceived weight matched the probabilistic cue. Each key-press altered the pull force of the linear (b) Weight perception task (WP)

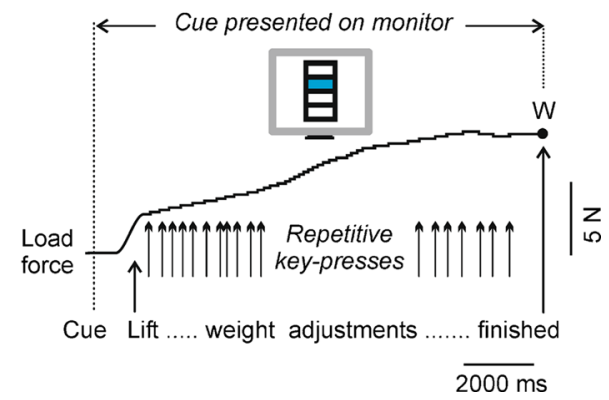

(b) In the weight perception task (WP), the object was lifted and its weight was adjusted by repetitive key-presses which operated the linear actuator (see Fig. 1a) until the perceived weight $\mathrm{W}$ matched the weight announced by the probabilistic cue. The cue (here $\mathrm{MH}$ ) was visible all the time. Note the different time scales in (a) and (b)

motor by $\sim 10 \mathrm{~g}(0.1 \mathrm{~N})$. The initial (preadjustment) weight varied between $250 \mathrm{~g}$ and $1550 \mathrm{~g}$. No time constraint was given, and it was allowed to lift and to put down/release the grip object while adjusting its weight. When the trial was completed, the space-bar was pressed, and the next cue was presented on the monitor (next trial). Each probabilistic cue was presented 10 times, resulting in 40 trials. The order of the cues was randomized. Each WP trial lasted 10-20 seconds; the entire task was $\sim 20$ minutes. The grasp configuration, which is known to affect perceived heaviness (Flanagan \& Bandomir, 2000), was the same in the GL and WP tasks.

\section{Data analysis}

Statistical analyses were performed using the $\mathrm{R}$ project for statistical computing (R Development Core Team, 2008; www.R-project.org.). For each probabilistic cue, the predicted object weight $\mathrm{P}$ was calculated as a weighted combination of the two possible weights, so cue HM $(66.7 \%$ heavy, $33.3 \%$ medium $)$ predicted a $\mathrm{P}$ of $1,200 \mathrm{~g}$ [ $(0.667 \mathrm{x}$ $1,400 \mathrm{~g})+(0.333 \times 800 \mathrm{~g})=1,200 \mathrm{~g}]$. The other $p$ values were $1,000 \mathrm{~g}$ for cue $\mathrm{MH}, 633 \mathrm{~g}$ for cue ML, and $467 \mathrm{~g}$ for cue LM. The $p$ values do not represent the subjects' individual expectations but indicate the statistically expected values for each cue, in line with research that applies statistical decision theory to motor behavior (Nagengast et al., 2010; Wolpert \& Landy, 2012).

GL task We analyzed trials in which the same actual weight (medium $=800 \mathrm{~g}$ ) was grasped and lifted, yet preceded by the four different probabilistic cues (LM, ML, MH, HM). No preprocessing of the data was performed. The first time derivatives of the grip and load forces, i.e., the grip force rate and load force rate (unit of measure: $\mathrm{N} / \mathrm{s}$ ), were calculated. Their maxima, the peak grip force rate (GFr) and peak load force rate (LFr), are reached before the object is lifted from its support (Fig. 2a; see Supplementary Figure 2 for raw data). These 
peak rates indicate anticipatory scaling of force output according to the weight that the subject expects to lift (Gordon et al., 1993; Nowak et al., 2013).

WP task The adjusted object weight W (unit of measure: gram), whose heaviness was perceived to match the prediction of a given probabilistic cue, was determined for each trial (Fig. 2b).

For each of the four probabilistic cues, subject-wise mean GFr and LFr and W values were calculated. To test for cuedependent changes of GFr, LFr, and W, analyses of variance (ANOVA) for repeated measurements with cue as withinsubject factor (4 levels) were performed on these subjectwise means. ANOVAs were calculated separately for groups A and B. We expected GFr, LFr, and W to increase with the predicted object weight $\mathrm{P}$. One-sided paired samples $t$ tests were calculated to test for a cue-dependent increase, with $p<0.0167$ after Bonferroni correction for multiple comparisons. For each contrast, the effect size was calculated, with $\mathrm{d}_{\text {cohen }}=(\mathrm{m} 1-\mathrm{m} 2) / \sqrt{ }\left(\mathrm{s} 1^{2}+\mathrm{s} 2^{2}\right) / 2$.

We plotted peak force rates (GFr, LFr) and $\mathrm{W}$ values against the weight $\mathrm{P}$ that was (statistically) predicted by the probabilistic cues (Figs. 3a and 4a). To compare the results of both tasks (GL and WP), subject-wise standardization (ztransformation) of the raw GFr, LFr, and W data were conducted. The mean values of the standardized weight adjustments (z-W) and peak force rates (z-GFr, z-LFr) were plotted against each other (Figs. $3 \mathrm{~b}$ and $4 \mathrm{~b}$, and Supplementary Figure 3). Overall correlations between the standardized results of the perceptual (WP) and motor (GL) tasks were described with Pearson's correlation coefficients $(r)$, and $t$ tests for significance of correlation were calculated. To examine the relation between the effects of the cues on weight adjustments and peak force rates paired $95 \%$ confidence regions were

(a)

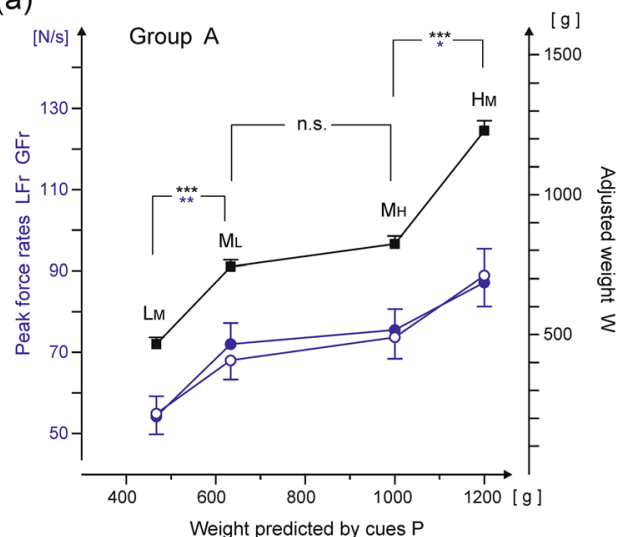

Fig. 3 Results of Group A (task sequence GL $\rightarrow$ WP). (a) Peak load force rates ( $\mathrm{LFr}$, filled blue circles), grip force rates (GFr, open blue circles) and adjusted weights $\mathrm{W}$ (black squares) increase with the weight $\mathrm{P}$ that the cues predicted (statistically). Means and SEM. Asterisks indicate significant cue-dependent differences (paired $t$-tests, $* p<.05$, ** $p<$ $.01, * * * p<.001$ after correction for multiple comparisons) for the tasks (GL, blue; WP, black). (b) Relation between z-transformed weight calculated for each of the four probabilistic cues, based on cue-wise correlation matrices, and illustrated (ellipsoids in Figs. 3 and 4). Finally, to test whether the distributions of $z-$ W and z-GFr (respectively z-LFr) differed, we conducted twosample Kolmogorov-Smirnov tests.

\section{Results}

Group A. The results of this group (task sequence GL $\rightarrow$ WP) are listed in Table 1 and illustrated in Fig. 3. The probabilistic cues influenced predictive force scaling during lifts of the medium weight $(800 \mathrm{~g})$ in the GL task. ANOVA indicated significant cue-dependent changes of the load force rate LFr $[F(3,30)=34.81, p<0.0001]$ and of the grip force rate $\mathrm{GFr}$ $[F(3,30)=18.82, p<0.0001]$. Significant cue-dependent changes $[F(3,30)=142.11, p<0.0001]$ also were found for the weights $(\mathrm{W})$ that were adjusted in the WP task. The two force rates (LFr, GFr) and the adjusted weight (W) generally increased as the (statistically) predicted weight $\mathrm{P}$ increased. However, these changes were significant only between cues LM and ML and between cues MH and HM, but not between cues ML and MH, as paired samples $t$ tests indicated (Table 1). Figure 3 a illustrates the resultant non-linear relations between the predicted weight $\mathrm{P}$ and the three variables (GFr, LFr, W), which increased less between cues ML and MH than between the other cues (LM and ML; MH and HM).

Figure $3 \mathrm{~b}$ shows the relationship between normalized ( $\mathrm{z}$ transformed) data of the perceptual (WP) and the motor task (GL) with the weight adjustments $(\mathrm{z}-\mathrm{W})$ and load force rates (z-LFr). Corresponding data (intrasubject averages) are depicted for each cue as points in a uniform color together with their attendant ellipsoid $95 \%$ confidence region. The confidence

(b)

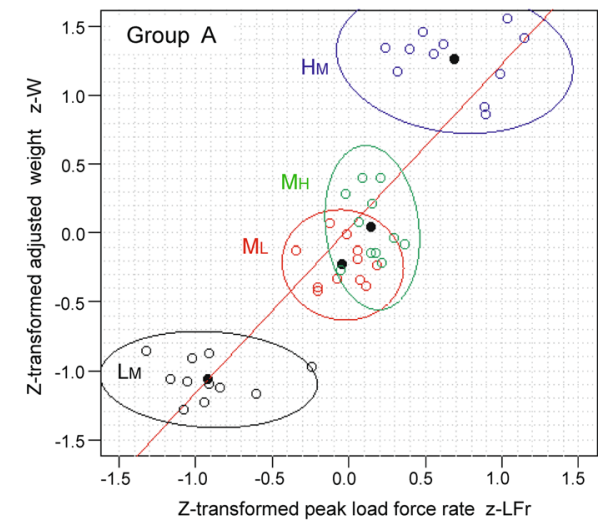

adjustments (z-W) and peak load force rates (z-LFr). Ellipsoids encircle the $95 \%$-confidence region of each cue; open symbols denote the subjectwise mean values, black dots the centers of the ellipsoids. Note that in (a) and (b) those trials are included where the same actual weight $(800 \mathrm{~g})$ was lifted, yet preceded by four different probabilistic cues (LM, ML, MH, HM). For a corresponding plot of $\mathrm{z}-\mathrm{W}$ versus z-GFr see supplementary figure III 
(a)

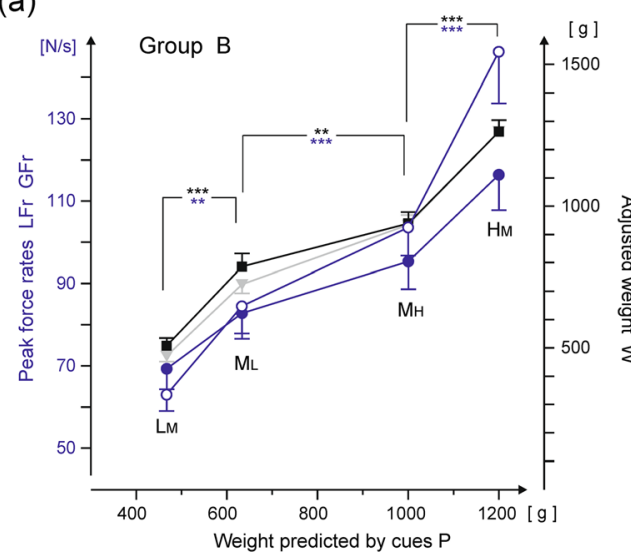

Fig. 4 Results of Group B (task sequence WP1 $\rightarrow$ GL $\rightarrow$ WP2). (a) Peak load force rates (LFr, filled blue circles), and grip force rates (GFr, open blue circles), and adjusted weights for the first (WP1: black squares) and the repeated weight perception task (WP2: grey lines with triangles). Error bars denote SEM. Asterisks indicate significant cue-dependent

regions of cues ML and MH overlap considerably, with their center points (black dots) lying in the overlapping region. Thus, cue-dependent differences of the load force rate were significant only between LM and ML and between MH and HM, but not between cues ML and MH. Equivalent results were obtained for the normalized grip force rate z-GFr (Supplementary Figure 3). Consequently, classification of the four cues resulted in three distinct relational categories between weight adjustment and predictive force scaling ( $\mathrm{LM}<\mathrm{ML} \approx \mathrm{MH}<\mathrm{HM}$ ) in group $\mathrm{A}$. Pearson's product-moment correlation revealed a high correlation between z-GFr and z-W, with $r=0.847, t(42)=10.31, p<$ $0.0001)$ and between $z-L F r$ and $z-W$, with $r=0.870, t(42)=$ $11.41, p<0.0001$. No significant differences between the distributions of z-GFr and z-W ( $p=0.316)$, as well as z-LFr and $z-\mathrm{W}$ (b)

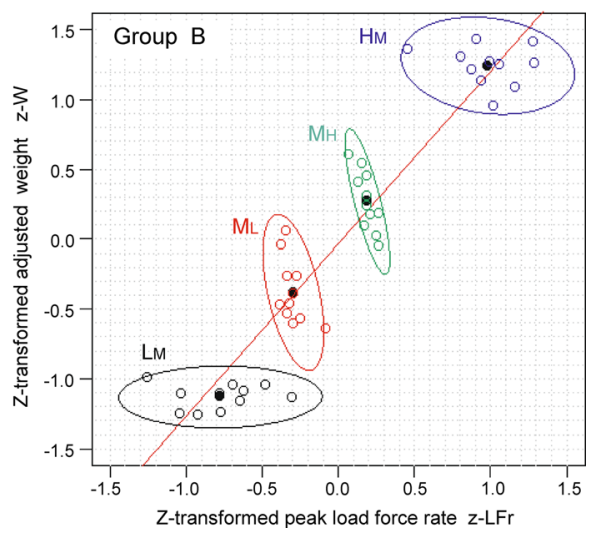

differences (paired $t$-tests, $* * p<.01, * * * p<.001$ after correction for multiple comparisons) (b) Relation between $z$-transformed weight adjustments (z-W) and peak load force rates (z-LFr). Otherwise as in Fig. 3. An analogous plot of z-W and z-GFr is shown in supplementary figure III

( $p=0.206)$ were found with Kolmogorov-Smirnov tests. Overall, a close correlation between the subject-wise mean values of the standardized weight adjustments and peak force rates was apparent.

Group B Data of this group (task sequence $\mathrm{WP}_{1} \rightarrow \mathrm{GL} \rightarrow$ $\mathrm{WP}_{2}$ ) are depicted in Fig. 4 and listed in Table 2. Exemplary original force curves are given in supplementary Figure 2. As in group A, ANOVA indicated significant cue-dependent changes of the force rates $\operatorname{LFr}[F(3,30)=65.90, p<0.001]$ and $\operatorname{GFr}[F(3,30)=8.84, p=0.0017]$ and also of the adjusted weights $\mathrm{W}_{1}\left[(F(3,30)=112.1, p<0.0001]\right.$ and $\mathrm{W}_{2}[F(3,30)=$ $152.2, p<0.0001]$ that had been set in the two WP tasks. All values increased significantly $(p<0.01$; Table 2$)$ in parallel

Table 1 Group A (order of tasks GL $\rightarrow$ WP): Effects of the probabilistic cues on predictive force scaling in the grip-lift task (GL) and on weight adjustments in the perceptual task (WP)

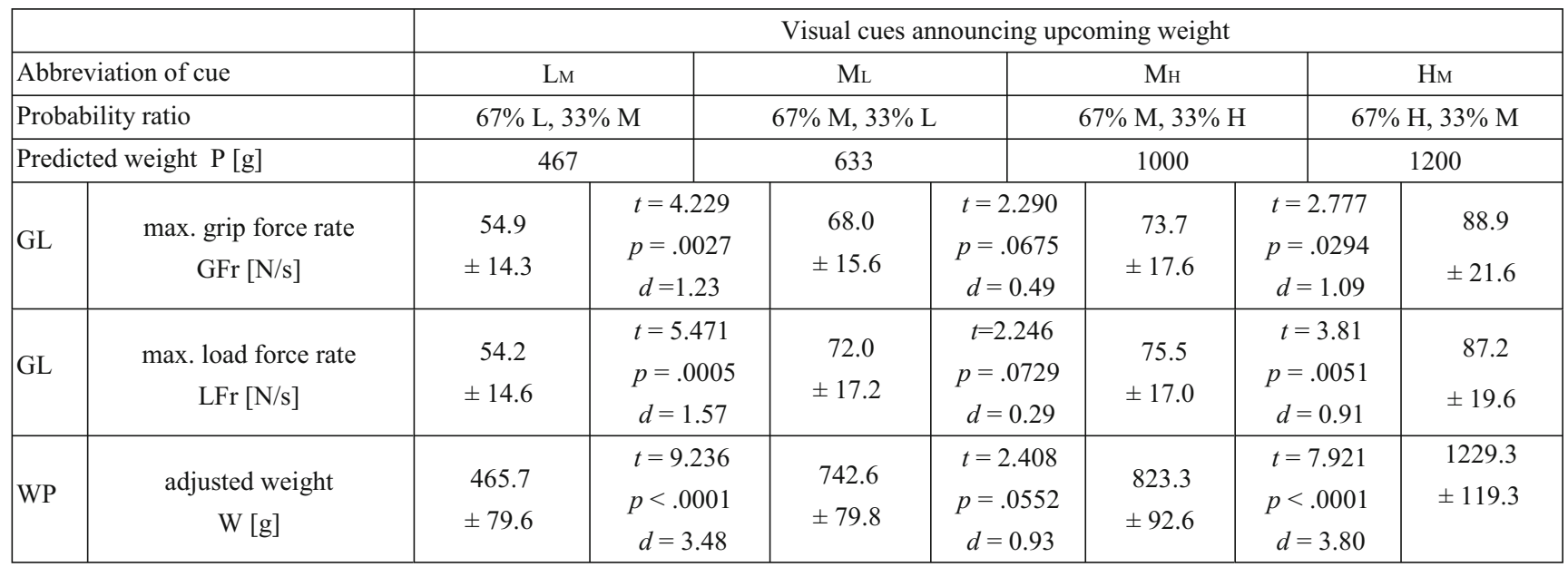

Means \pm standard deviations of the individual means of group A ( $\mathrm{n}=11$ subjects; with 10-20 trials per probabilistic cue in each subject). $t$ values and $p$ values (Bonferroni corrected for multiple comparisons) of one-sided paired samples $t$ tests (post ANOVA; 10 degrees of freedom) comparing the values of adjacent columns. $d=$ effect size $\mathrm{d}_{\text {cohen }}$ 
Table 2 Group B (order of tasks $\mathrm{WP}_{1} \rightarrow \mathrm{GL} \rightarrow \mathrm{WP}_{2}$ ): Effects of the probabilistic cues on predictive force scaling in the grip-lift action (GL) and on weight adjustments in the perceptual task $\left(\mathrm{WP}_{1,2}\right)$

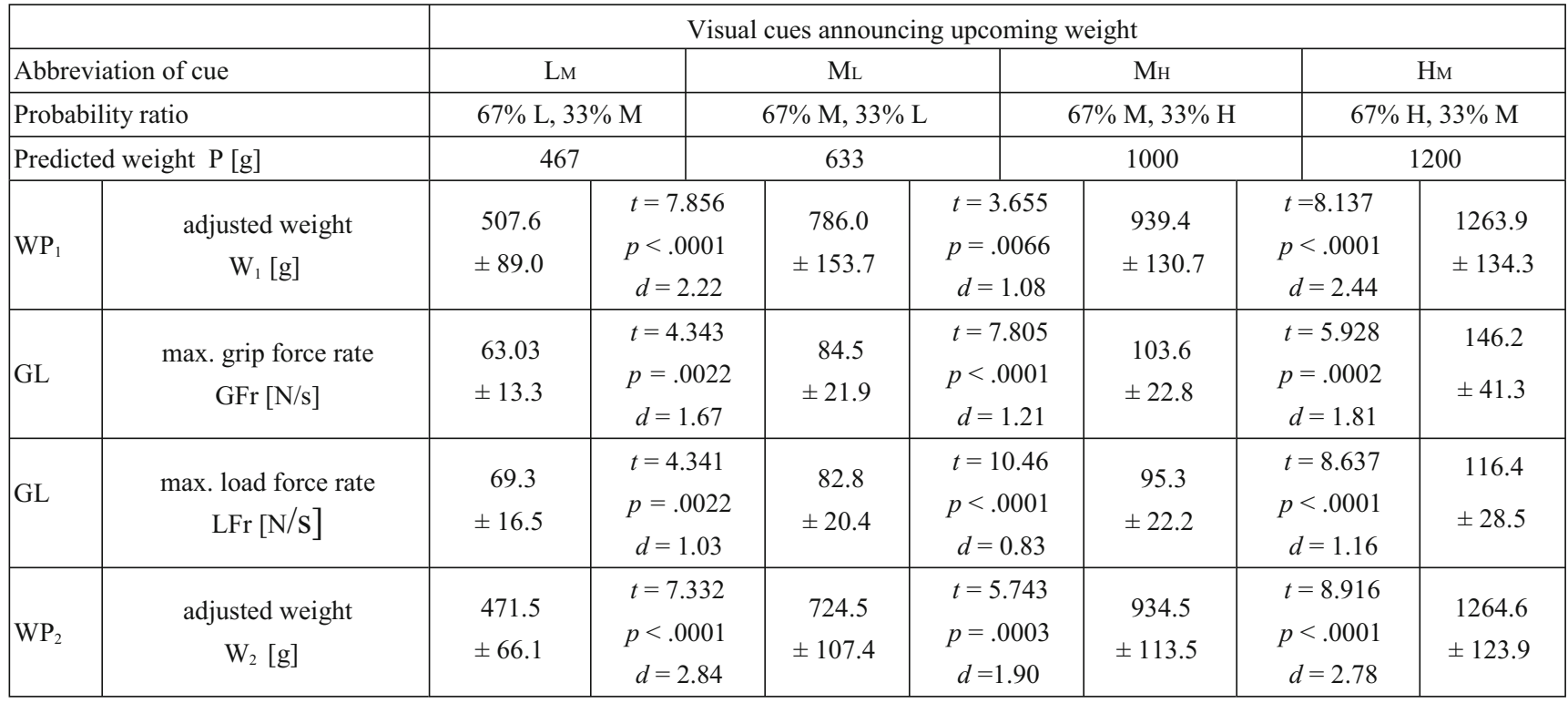

Means \pm standard deviations of the individual means of group $B$ ( $n=11$ subjects; with 10-20 trials per probabilistic cue in each subject). The perceptual task was performed twice $\left(\mathrm{WP}_{1}, \mathrm{WP}_{2}\right) . t$ values and $p$ values (Bonferroni corrected for multiple comparisons) of one-sided paired samples $t$ tests (post ANOVA; 10 degrees of freedom) comparing the values of adjacent columns. $d=$ effect size $\mathrm{d}_{\text {cohen }}$

with the predicted object weight $\mathrm{P}(\mathrm{LM}<\mathrm{ML}<\mathrm{MH}<\mathrm{HM})$. In contrast to group $\mathrm{A}$, the differences between cues $\mathrm{ML}$ and MH were now significant, i.e., LFr, GFr, and $\mathrm{W}$ values were now clearly higher after presentation of cue MH than after cue ML (Fig. 4a; Table 2 for $p$ and $t$ values). We found no significant differences between the first $\left(\mathrm{W}_{1}\right)$ and the second $\left(\mathrm{W}_{2}\right)$ weight adjustment experiments $[F(1,10)=0.14, p=0.71)]$.

Figure $4 \mathrm{~b}$ depicts the relation between weight adjustments $(\mathrm{z}-\mathrm{W})$ and predictive load force scaling (z-LFr) in group B with normalized data and $95 \%$ confidence regions (ellipsoids) for each cue. The results of the normalized grip force rate (z-GFr) and $z-W$ are very similar (Supplementary Figure 3). The confidence regions of the cues show no overlap in group B, for both, the normalized load force rate (z-LFr, Fig. 4b) and grip force rate (z-GFr) in relation to the weight adjustments $(z-W)$. Unlike in group $\mathrm{A}$, the differences were now significant between all cues. Consequently, classification of the four cues resulted in four distinct categories of weight adjustments and predictive scaling of the load and grip forces. Again there was a high correlation between z-LFr and z-W, with $r=0.936, t(42)=$ 17.229, $p<0.0001$; and also between $\mathrm{z}-\mathrm{GFr}$ and $\mathrm{z}-\mathrm{W}$, with $r=0.934, t(42)=16.976, p<0.0001$. Kolmogorov-Smirnov tests did not disclose any significant differences between the distributions of z-GFr and z-W ( $p=0.6336)$, and z-LF and z-W ( $p=0.3161)$, respectively. The mean differences between groups A and B are listed in a supplementary table.

To analyze the impact of the cues ML and MH on participants' peak force rates (GFr and LFr) across the two groups in more detail, we tested for possible interactions between group and cue on peak force rates with a post-hoc mixed withinbetween 2 x 2 ANOVA (cues: ML, MH; groups: A, B). There were significant interactions between cue and group affiliation, for both, $\operatorname{LFr}[F(1,20)=21.53, p<0.001]$ and $\operatorname{GFr}[F(1,20)=14.39, p=0.0012]$. A further post-hoc $2 \times 2$ ANOVA indicated such an interaction also for the adjusted weights $[F(1,20)=6.79, p=0.0017]$.

All in all, the results indicated 1) effects of the probabilistic cues on predictive force scaling (LFr and GFr) in the grip-lift task and on weight adjustment (W) in the perceptual task, 2) a high correlation between the standardized results of both tasks (z-LFr and z-W; z-GFr and z-W) without significant differences between their distributions, and 3 ) an influence of the task sequence (group A vs. group B) that resulted in a different implementation of the probabilistic information in predictive force scaling and weight adjustment, depending on the task that was performed first.

\section{Discussion}

We have devised a novel paradigm to study how the perceptual and the motor system deal with explicit probabilistic cues to an object's weight. Two groups of participants accomplished a grip-lift (GL) and a weight perception (WP) task in different order. In the GL task, the participants should utilize four probabilistic cues (LM, ML, MH, HM) to initiate their grip-lift actions with appropriately scaled grip and lift force rates. In line with a previous study (Trampenau et al., 2015) we analyzed 
trials where the same actual weight (medium $=800 \mathrm{~g}$ ) was grasped and lifted, yet preceded by the four different probabilistic cues. These cues also were presented in the WP task, where participants gradually adjusted the weight of an object, with continuous proprioceptive and tactile feedback, until the heaviness matched the expectation for a given probabilistic cue. Three aspects are noteworthy, namely an effect of the task sequence, high correlations and similar distributions of the standardized results of both tasks, and distortion of the probabilistic information of the cues.

As a sequence effect, the initial task determined handling of the probabilistic information in the whole experiment. Members of group A started with the GL task, where they experienced three different weights that were actually lifted. Moreover, as a consequence of our paradigm, the frequency of the medium weight was 1.75 times higher than the frequencies of the other two weights across all trials of the GL task. Consequently, the four probabilistic cues were related to three distinct categories of peak force rates ( $\mathrm{LM}<\mathrm{ML} \approx \mathrm{MH}<\mathrm{HM}$ ), indicating three different modes of action initiation. In the subsequent WP task, the same four cues were again assigned to three categories of heaviness, so that the initially established implementation of the probabilistic information was maintained. Conversely, the four cues were associated with four different weights ( $\mathrm{LM}<\mathrm{ML}<\mathrm{MH}<\mathrm{HM}$ ) when the WP task was performed before the GL task (group B), in accordance with stepwise increases of the statistically predicted weight $P$. Anticipatory scaling of the force rates in the subsequent GL task then replicated the same pattern of four distinct predictions (LM $<\mathrm{ML}<\mathrm{MH}<\mathrm{HM}$ ), which again became evident during a final repetition of the WP task.

During the WP task, the subjects had ample time to adjust the object's weight and heaviness while considering the probabilistic information of the cue; up to 20 seconds were needed for each trial. Selection of appropriate weights during the WP task, when performed first, was based on abstract knowledge of the probabilities, and thus involved a decision from description according to the terminology of Hertwig, Barron, Weber, and Erev (2004). In the GL task, a cue was presented for just a second and then the grip-lift was initiated, so that the probabilistic information was implemented without long hesitation. Because the subjects encountered the three different weights more or less often during the series of 150 trials, the choice of an appropriate force rate was at least in part a decision from experience (Hau, Pleskac, \& Hertwig, 2010; Hertwig et al., 2004). Despite the different nature of both tasks, their results were highly correlated, following the pattern that had been established during the task performed first (see above). This correlation adds evidence to a close connection between perception and action in our experiment, perhaps in line with the more general theory that perception and action rely on common abstract concepts (Franz, Gegenfurtner, Bülthoff, \& Fahle, 2000; Müsseler, 1999; Prinz, 1990). Similar assumptions were also formulated for social behavior in the theory of intentional schemas (Barresi \& Moore, 1996), and in the motor theory of speech perception (Liberman \& Mattingly, 1985).

Also, previous research found associations between perceptual weight estimates and scaling of the fingertip forces. If more grip force is needed to grasp an object due to a lower friction (smooth surface texture) or because the contact area between fingers and object is small, then the object is perceived to be heavier (Flanagan, Wing, Allison, \& Spenceley, 1995; Flanagan \& Bandomir, 2000). Van Polanen and Davare (2015) recently demonstrated that if there is a mismatch between expected and actual object weight in a grip-lift task, the execution of force corrections will influence weight perception in a proportional manner. Still, action and perception can dissociate under certain conditions. Experiments with optical illusions have shown that perceived illusory cues to an object's size are not matched by motor parameters such as the aperture of the hand during prehension (Aglioti, DeSouza, \& Goodale, 1995), which conforms to the dual-stream theory of the visual system proposed by Goodale \& Milner (1992). A material-weight illusion occurs when subjects lift objects of identical mass and size that are coated with lighter- or heavier-looking material (e.g., wood and metal), so that their actual mass differs from the expectation for the particular material (Ellis \& Lederman, 1999). Objects made from light-looking material feel heavier than objects made from heavy-looking material, although the grip and load forces are scaled to the actual identical weight after several grip-lift trials (Buckingham, Cant, \& Goodale, 2009). The classical size-weight illusion takes place when two objects of equal mass but different sizes are lifted (Charpentier, 1891). The smaller object is perceived to be heavier, although again the fingertip forces are scaled to its actual weight after several grip-lift trials (Buckingham \& Goodale, 2010; Flanagan \& Beltzner, 2000). Judgements of perceived heaviness in experiments with these illusions typically involve numerical rating scales or a forced choice to decide which object feels heavier. It might be interesting to examine whether such judgements can be replicated with a WP task, where the weight of a neutral object (or handle, etc.) is adjusted so that its heaviness matches the effect of the illusion. If this was the case, then results of the WP task (load force reflecting the effect of the illusion) and of the GL task (force is scaled to the actual object weight) would indeed dissociate. The paradigm of the present study, however, did not evoke a mismatch between perception and action; the probabilistic information was implemented in a similar way during both tasks (WP and GL).

In accordance with our previous report (Trampenau et al., 2015), we found a nonlinear relationship between the peak force rates and the statistically predicted object weight $P$, in particular in group A who started with the GL task. For each cue, we calculated the predicted object weight $\mathrm{P}$ as a weighted combination of the two possible weights. This corresponds 
with research that views motor control as decision-making and applies equations that formalize decisions in economics, which are typically modeled as a choice among lotteries (Nagengast et al., 2010; Wolpert \& Landy, 2012; Wu et al., 2009). The expected value of a lottery is the sum of the values weighted by the corresponding probabilities, so that a 50:50 gamble of winning either $\$ 100$ or $\$ 10$ results in an expected gain of $\$ 55$. The predicted weight $\mathrm{P}$ was calculated in the same way. Still, how are these $p$ values related to the subjects' performance? Members of group A started with the GL task, i.e., the initial task that determined the interpretation of the probabilistic information comprised repetitive grip-lift actions. Of the many possible policies to utilize $p$ values for a selection of appropriate force rates, three hypothetical strategies are outlined, each representing an extreme option.

One theoretical option (a) may be to disregard all probabilistic cues and associated $p$ values and instead to always scale the forces to the medium weight, which turns up most often (70/ 150 trials). This strategy would be associated with frequent large errors of initial force scaling and is incompatible with the demonstrated effects of the cues on grip and load force dynamics. Another strategy (b) could always scale the force rates to the current predicted weight $\mathrm{P}$, resulting in a linear relation between the $p$ values and peak force rates. Across all GL trials, the sum of all positive deviations ("actual weight is heavier than P") with ensuing corrections will then equal the sum of all negative mismatches ("actual weight is lighter than $\mathrm{P}$ "). Consider $\Delta \mathrm{W}$ as the difference between the two possible weights that follow a cue, e.g., $\Delta \mathrm{W}=1400 \mathrm{~g}-800 \mathrm{~g}=600 \mathrm{~g}$ for cue $\mathrm{MH}$. The actual weight will be $(0.67 \Delta \mathrm{W})$ heavier than $\mathrm{P}$ in one third of all $\mathrm{MH}$ trials and $(0.33 \Delta \mathrm{W})$ lighter than $\mathrm{P}$ in the other two thirds of such trials. Analogous calculations pertain to the other cues; the sum of all signed deviations from the targeted weight $P$ will always be zero. Strategy (b) avoids large errors of initial force scaling and repetitive unidirectional corrections of the preprogramed forces, which may be beneficial.

A third strategy (c) may always aim for the more likely weight, i.e. treat the higher probability $(66.7 \%)$ like a certain prediction (100\%). In two thirds of all trials, preprograming will then be optimal because a given cue is actually followed by the more likely weight, but in one third of all trials, large initial force over- or undershoots will occur, because the participant has aimed for the "wrong" weight (deviation $=\Delta \mathrm{W}$ ). As an advantage of this strategy, the sum of the absolute values of all deviations across a series of $\mathrm{n}$ trials will be minimal, amounting to $[0.33 \mathrm{n}(|\Delta \mathrm{W}|)]$. In other words, the sum of all mismatches and subsequent corrections, regardless of their direction, will be smaller than with any other policy. Strategy (c) will bring forth a clearly nonlinear relation between the force rates and the $\mathrm{P}$ values, because cues $\mathrm{MH}$ and $\mathrm{ML}$ are equivalent; both indicate $66.7 \%$ probability of the medium weight.

Our results suggest that members of group A, who started with the GL task, did not clearly differentiate between cues
ML and MH, as evident from the similar peak force rates and, correspondingly, similar weight adjustments during the subsequent WP task (Fig. 3a; Table 1). This is in line with strategy (c), perhaps attenuated by a tendency to avoid very large initial errors, as the force rate maxima were not exactly identical for cues ML and MH. In other words, cues indicating a low probability (33.3\%) of a weight differing from the medium value had much smaller influence on force scaling than cues predicting a high likelihood (66.7\%) of such a difference, confirming our previous results (Trampenau et al., 2015). Distortion of probabilistic information also has been found in other motor paradigms, such as a rapid-pointing task that involved decisions under risk (Wu et al., 2009). Subjects underweighted small probabilities and overweighted large probabilities, as the participants in our experiment did.

For members of group B the initial experiment, which determined the interpretation of the probabilistic information, was the WP task. In principle, members of group B could use the aforementioned strategies to interpret the cues during their weight adjustments. Certainly with the difference that they could fine-tune the weight until the proprioceptive and tactile feedback matched their expectation for a given cue. Weight adjustments that match up P values would adhere to the explicitly known probabilities $(33.3 \%, 66.6 \%)$ and thus represent the average weight across a series of grip-lift trials following a given cue. In line with this, subjects of group B associated cues ML and MH with two different weights during the initial WP task and, correspondingly, then applied dissimilar force rates during the GL task (Fig. 4a; Table 2). Still, the relationship between the force rates and the $\mathrm{P}$ values did not appear perfectly linear. One might interpret this pattern as a compromise, where the subjects avoid large errors of initial force scaling by considering the different predictions of cues MH and ML [strategy (b)], but at the same time aim towards the more likely medium weight, which is in line with an influence of strategy (c).

Concerning the balance between different internal models of object properties, another explanation can be deduced from the report of Davidson and Wolpert (2004), who studied a GL task where two familiar objects were combined by stacking them and then lifted. Predictive force scaling during the initial trials was not entirely based on an addition of the two wellknown weights in their study but biased by a default weight estimate for all possible combined weights. In our experiment, the participants therefore may have combined different internal models of object dynamics, which were based on probabilistic information given by the current cue, on an average estimation (default) of all weights, and on a general strategy of utilizing probabilistic information, which had been established during the initial task (GL or WP).

The present study has limitations. A detailed analysis of the relationship (exact shape of the function) between predicted weights $\mathrm{P}$ and the force rates would require a finer gradation, i.e., further probabilistic conditions. It might be interesting to 
know whether a different mode of cue presentation (shape, arrangement) can influence the results. Our apparatus did not precisely model the way freely moveable objects would respond to lift forces. The linear actuator provided a constant force during each GL trial, and the object, i.e. the handle with the force transducers, was lifted along a predetermined movement path. Yet the original force curves (Supplementary Figure 2) resemble data that were recorded with real objects. Moreover, our analysis was based on the force rate peaks, which were typically reached before lift-off, before the slightly unnatural lifting movement took place. Experiments with multiple real objects may be an issue of future research.

In summary, normal volunteers performed a perceptual task and a motor task that both involved the interpretation and utilization of four probabilistic cues to an object's weight. The results of both tasks were highly correlated, suggesting that an overarching concept ruled the implementation of the probabilistic information during weight perception and action (grip-lift). This concept was established during the task that was performed first, as evident from the effect of the sequence of the tasks. If the perceptual task was performed first, the four probabilistic cues were related to four distinct categories of heaviness. If the motor task was performed first, the same four cues were assigned to three different categories, corresponding to the experience that three different weights were actually offered after the four cues. Interpretation of probabilistic information therefore was not fixed but adapted to the demands of the initial task. The first experience thus had a significant influence on the categorization of the cue-related information. In a more general sense, the initial strategy, once established, determines the way probabilistic information is interpreted and used.

Acknowledgements The authors thank Martin Müller for construction of the mechanical experimental set-up, Franz Faul for his outstanding methodical and theoretical contribution, Nina Lau for her helpful comments, and Kirsten Zeuner for her support.

\section{References}

Aglioti, S., DeSouza, J. F. X., \& Goodale, M. A. (1995). Size-contrast illusions deceive the eye but not the hand. Current Biology, 5, 679-685.

Ameli, M., Dafotakis, M., Fink, G. R., \& Nowak, D. A. (2008). Predictive force programming in the grip-lift task: The role of memory links between arbitrary cues and object weight. Neuropsychologia, 46(9), 2383-2388.

Barresi, J., \& Moore, C. (1996). Intentional relations and social understanding. Behavioral and Brain Sciences, 19, 107-129.

Baugh, L. A., Kao, M., Johansson, R. S., \& Flanagan, J. R. (2012). Material evidence: Interaction of well-learned priors and sensorimotor memory when lifting objects. Journal of Neurophysiology, 108, $1262-1269$.

Buckingham, G., Cant, J. S., \& Goodale, M. A. (2009). Living in a material world: How visual cues to material properties affect the way that we lift objects and perceive their weight. Journal of Neurophysiology, 102, 3111-3118.

Buckingham, G., \& Goodale, M. A. (2010). Lifting without seeing: The role of vision in perceiving and acting upon the size weight illusion. PLoS ONE, 5(3), e9709.

Charpentier, A. (1891). Analyse expérimentale: De quelque éléments de la sensation de poids. Archives de Physiologie Normale et Pathologique, 3, 122-135.

Chouinard, P. A., Large, M. E., Chang, E. C., \& Goodale, M. A. (2009). Dissociable neural mechanisms for determining the perceived heaviness of objects and the predicted weight of objects during lifting: An fMRI investigation of the size-weight illusion. Neuroimage, 44, 200-212.

Chouinard, P. A., Leonard, G., \& Paus, T. (2005). Role of the primary motor and dorsal premotor cortices in the anticipation of forces during object lifting. Journal of Neuroscience, 25(9), 2277-2284.

Cole, K. J., \& Rotella, D. L. (2002). Old age impairs the use of arbitrary visual cues for predictive control of fingertip forces during grasp. Experimental Brain Research, 143, 35-41.

Davidson, W. R., \& Wolpert, D. M. (2004). Internal models underlying grasp can be additively combined. Experimental Brain Research, 155(3), 334-340.

Development Core Team, R. (2008). R: A language and environment for statistical computing. Vienna: R Foundation for Statistical Computing. https://www.r-project.org

Ellis, R. R., \& Lederman, S. J. (1999). The material-weight illusion revisited. Perception \& Psychophysics, 61, 1564-1576.

Flanagan, J. R., \& Bandomir, C. A. (2000). Coming to grips with weight perception: Effects of grasp configuration on perceived heaviness. Perception \& Psychophyics, 62, 1204-1219.

Flanagan, J. R., \& Beltzner, M. A. (2000). Independence of perceptual and sensorimotor predictions in the size-weight illusion. Nature Neuroscience, 3, 737-741.

Flanagan, J. R., Wing, A. M., Allison, S., \& Spenceley, A. (1995). Effects of surface texture on weight perception when lifting objects with a precision grip. Perception \& Psychophyics, 57, 282-290.

Franz, V. H., Gegenfurtner, K. R., Bülthoff, H. H., \& Fahle, M. (2000). No evidence for a dissociation between perception and action. Psychological Science, 11, 20-25.

Goodale, M. A., \& Milner, A. D. (1992). Separate visual pathways for perception and action. Trends in Neurosciences, 15, 20-25.

Gordon, A. M., Westling, G., Cole, K. J., \& Johansson, R. S. (1993). Memory representations underlying motor commands used during manipulation of common and novel objects. Journal of Neurophysiology, 69, 1789-1796.

Grandy, M. S., \& Westwood, D. A. (2006). Opposite perceptual and sensorimotor responses to a size-weight illusion. Journal of Neurophysiology, 95, 3887-3892.

Hau, R., Pleskac, T. J., \& Hertwig, R. (2010). Decisions from experience and statistical probabilities: Why they trigger different choices than a priori probabilities. Journal of Behavioral Decision Making, 23, 48-68.

Hertwig, R., Barron, G., Weber, E. U., \& Erev, I. (2004). Decisions from experience and the effect of rare events in risky choice. Psychological Science, 15, 534-539.

Jenmalm, P., Schmitz, C., Forssberg, H., \& Ehrsson, H. H. (2006). Lighter or heavier than predicted: Neural correlates of corrective mechanisms during erroneously programmed lifts. Journal of Neuroscience, 26(35), 9015-9021.

Johansson, R. S., \& Flanagan, J. R. (2009). Coding and use of tactile signals from the fingertips in object manipulation tasks. Nature Reviews Neuroscience, 10, 345-359.

Johansson, R. S., \& Westling, G. (1984). Roles of glabrous skin receptors and sensorimotor memory in automatic-control of precision grip when lifting rougher or more slippery objects. Experimental Brain Research, 56, 550-564. 
Johansson, R. S., \& Westling, G. (1988). Coordinated isometric muscle commands adequately and erroneously programmed for the weight during lifting task with precision grip. Experimental Brain Research, 71(1), 59-71.

Liberman, A. M., \& Mattingly, I. G. (1985). The motor theory of speech perception revised. Cognition, 21, 1-36.

Müsseler, J. (1999). How independent from action control is perception? An event-coding account for more equally-ranked crosstalks. In G. Aschersleben, T. Bachmann, \& J. Müsseler (Eds.), Cognitive contributions to the perception of spatial and temporal events (Advances in Psychology (Vol. 129, pp. 121-147). Amsterdam: Elsevier.

Nagengast, A. J., Braun, D. A., \& Wolpert, D. M. (2010). Risk-sensitive optimal feedback control accounts for sensorimotor behavior under uncertainty. PLoS Computational Biology, 6(7), e1000857.

Nowak, D. A., Glasauer, S., \& Hermsdörfer, J. (2013). Force control in object manipulation-a model for the study of sensorimotor control strategies. Neuroscience \& Biobehavioral Reviews, 37, 1578-1586.

Nowak, D. A., \& Hermsdörfer, J. (2005). Grip force behavior during object manipulation in neurological disorders: Toward an objective evaluation of manual performance deficits. Movement Disorders, 20, $11-25$.

Prinz, W. (1990). A common coding approach to perception and action. In O. Neumann \& W. Prinz (Eds.), Relationships between perception and action (pp. 167-201). Berlin: Springer-Verlag.

Trampenau, L., Kuhtz-Buschbeck, J. P., \& van Eimeren, T. (2015). Probabilistic information on object weight shapes force dynamics in a grip-lift task. Experimental Brain Research, 233, 1711-1720.

Van Polanen, V. \& Davare, M. (2015). Sensorimotor Memory Biases Weight Perception During Object Lifting. Frontiers in Human Neuroscience 2015, 9, Article 700.

Wolpert, D. M., \& Landy, M. S. (2012). Motor control is decision-making. Current Opinion in Neurobiology, 22, 996-1003.

Wu, S. H., Delgado, M. R., \& Maloney, L. T. (2009). Economic decisionmaking compared with an equivalent motor task. Proceedings of the National Academy of Sciences, 106(15), 6088-6093.

Zhang, H., \& Maloney, L. T. (2012). Ubiquitous log odds: A common representation of probability and frequency distortion in perception, action, and cognition. Frontiers in Neuroscience, 6, 1-14. 